\title{
Considerações sobre a disponibilidade dos tópicos de Astronomia em livros didáticos de Física do PNLD $2018^{+*}$
}

Gustavo Fontoura Souza ${ }^{1}$

Instituto Federal de Educação, Ciência e Tecnologia do Rio Grande do Norte Parnamirim - RN

Janilson Simões de Azevedo Filho ${ }^{1}$

Escola Estadual Filomena de Azevedo

Santo Antônio - RN

\section{Resumo}

O presente trabalho relata as análises realizadas nos livros didáticos de Física do Ensino Médio, aprovados e ofertados pelo Programa Nacional do Livro Didático (PNLD), para o triênio2018-2020, acerca da abordagem dos conhecimentos sobre Astronomia. A pesquisa bibliográfica também consultou o PCN + de Ciências Naturais para o Ensino Médio, que possui como um dos seus temas estruturadores o "Universo, Terra e Vida”, para o diagnóstico da relação entre os conteúdos e temas abordados nesse com os contemplados dentro da estrutura dos livros didáticos analisados. A pesquisa é considerada de natureza quantitativa, embasada em revisão bibliográfica de obras que versam tanto sobre o Ensino de Física quanto da Astronomia, além de apresentar referências e orientações oficiais sobre as propostas curriculares para o trabalho pedagógico das ciências naturais no Ensino Médio, última etapa da Educação Básica. Na análise das informações coletados em cada obra, como também na comparação entre as coleções que foram objetos de estudo deste trabalho de pesquisa, constata-se que as obras disponibilizadas pelo PNLD 2018 não são consonantes em relação à quantidade de assuntos astronômicos abordados, considerando como referencia as orientações específicas do PCN + de Ciências Naturais para o Ensino Médio.

\footnotetext{
${ }^{+}$Analysis of the Astronomy knowledge approach in PNLD Physics books 2018

* Recebido: abril de 2020. Aceito: julho de 2020.

${ }^{1}$ E-mails: gfontoura@gmail.com; janilson.fisica@hotmail.com
} 
Palavras-chave: Astronomia; Física; Livro Didático.

\begin{abstract}
This work presents the results of the analysis of High School Physics textbooks, approved and offered by the "Programa Nacional do LivroDidático" (PNLD), for the triennium 2018-2020, about the way they approach and present knowledge about Astronomy. The bibliographic research also consulted the PCN + of Natural Sciences for Secondary Education, which has as one of its themes the "Universe, Earth and Life", for the diagnosis of the relationship between the contents and themes addressed in that and those contemplated within structure of the textbooks analyzed. The research is considered of a qualitative nature, based on a bibliographical review of works that deal with both Physics and Astronomy teaching, as well as presenting official references and guidelines on the curricular proposals for the pedagogical work of the natural sciences in High School, the last stage of Basic Education. In the analysis of the data collected in each work, as well as in the comparison between the collections that were objects of study of this research, it is verified that the works made available by the PNLD 2018 are not consonant in relation to the amount of astronomical subjects approached, according to the specific guidelines of the PCN + of Natural Sciences for High School.
\end{abstract}

Keywords: Astronomy; Physics; Textbooks.

\title{
I. Introdução
}

O ser humano, desde os primórdios de sua existência no planeta, vem observando e deslumbrando-se com os belos e intrigantes fenômenos que ocorrem na natureza, fascinandose a cada deleite de suas belezas. Tudo que ocorria ao entorno do ser primitivo era digno de sua atenção, desde um simples nascimento de uma planta até os raios e trovões oriundos dos choques das nuvens. E como não deixaria de ser, o céu era um dos principais focos de admiração, devido aos acontecimentos intrigantes acometidos no mesmo, os objetos que o compõe e o "mistério" escondido em sua beleza. Amaral (2008) faz referência ao ser histórico, vinculando a Astronomia à formação sociocultural de várias culturas e seus sujeitos.

A partir dessas observações e curiosidades sobre o céu, muitas questões provocantes foram levantadas: o que realmente são esses acontecimentos que se enredam na abóboda celeste? Do que eles são constituídos ou são gerados? Quais os propósitos que permitem suas existências? Quais são as funções deles? 
Diversos filósofos e cientistas consideram a Astronomia como a primeira ciência criada pelo homem com conhecimentos sistemáticos, subsidiando (em muitos casos) as primeiras investigações científicas da Matemática. A humanidade, da Pré-História até os dias atuais observam e desvendam o céu, a fim de guiarem seus passos e destinos.

A partir de diversas indagações relativas à astronomia e a importância desse conhecimento para a humanidade, o ensino de Astronomia começou a ser implementado, tanto no ensino formal quanto no ensino informal. No Brasil o primeiro curso de astronomia foi criado em 1958, na antiga Universidade do Brasil (Langhi e Nardi, 2009). Contudo, após diversas reformas do ensino, os Parâmetros Curriculares Nacionais incluíram conteúdos da astronomia na disciplina de ciências, no ensino fundamental e de física, no ensino médio (Brasil, 2019).

Existem diversas justificativas para a inclusão do ensino de Astronomia no ensino formal. Langhi e Nardi (2014) elencam alguns aspectos relativos a essas justificativas considerando trabalhos publicados por pesquisadores. Dentre estas justificativas, destacamos os fatos de que a astronomia é um elemento interdisciplinar e motivador de atividades que favorece a elaboração de atividades experimentais e a prática observacional do céu (Langhi e Nardi, 2014). Nesse contexto, entende-se que o ensino de astronomia na educação básica, permite atiçar a curiosidade do aluno e proporcionar a realização de atividades práticas e envolver outras disciplinas nessas atividades criando um ambiente interdisciplinar e investigativo.

De acordo com o Ministério da Educação, o livro didático é uma das principais formas de documentações e de consulta para professores e alunos no país, chegando, às vezes, a "influenciar o trabalho pedagógico e o cotidiano da sala de aula" (BRASIL, 2003). Em alguns casos, entre as mais diversas condições de ensino e aprendizagem que existem no Brasil, o professor de Ciências tem no livro didático s sua única fonte de consulta. Isso quer dizer que, para alguns o livro didático é uma ótima fonte de informações e aprendizado, para outros é a única. Diante disso, é fundamental que o livro didático, sobretudo o que é utilizado na rede pública do país, precisa contemplar os conteúdos definidos nos marcos regulatórios e nas legislações pertinentes, sob pena de privar alunos do acesso ao conhecimento.

Dessa forma, o presente trabalho se propõe, através de revisões bibliográficas de materiais didáticos e leituras dos documentos oficiais, avaliar a disponibilidade de tópicos de Astronomia nos livros de Física oferecidos às escolas públicas que ofertam o Ensino Médio, última modalidade da Educação Básica. Com isso, expõe-se pontos positivos e lacunas presentes nas obras ofertadas pelo PNLD 2018 sobre conhecimentos e trabalhos de pesquisa relacionados ao cosmos, conforme os pressupostos presentes nas orientações curriculares. 


\section{Revisão bibliográfica}

\section{II.1 O Ensino de Astronomia}

No campo de pesquisa e ensino cada dia mais ampliado e diverso encontrado no âmbito das Ciências da Natureza, a Astronomia pode ser considerada um dos mais belos e atraentes estudos a serem abordados. Dentro dos ambientes educacionais, abordagem de conteúdos astronômicos não atende apenas as recomendações das orientações presentes nos documentos oficiais. A Astronomia, com seus encantos e mistérios, tem a capacidade de atrair a atenção e facilitar as aprendizagens dos conteúdos das ciências naturais

$\mathrm{O}$ ensino de ciências naturais possibilita aos alunos a enxergarem com mais objetividade os fenômenos acometidos na natureza que lhes rodeia. Segundo observações de Langhi e Nardi (2014), quando se coloca os discentes em situações facilitadoras de aprendizagem, em que são convidados a questionarem e analisarem criteriosamente algo que ocorre no ambiente onde se localiza, ou características peculiares e intrigantes de um elemento natural, eles podem apreender com mais facilidade os conhecimentos e saberes esperados. Langhi e Nardi (2014) também citam o elemento motivador da Astronomia, destacando que os conhecimentos astronômicos possibilitam o despertar do interesse pelo estudo de ciências naturais, além do fascínio e a curiosidade pelos estudos da ciência celeste.

Freire (1996) intenta para a importância que a ação didática deve assumir em relação a curiosidade no processo de ensino, colocando-a como uma inquietação que contribui para a construção de conhecimento e/ou esclarecimento de algo. Dessa forma, a curiosidade é um elemento necessário para que ocorra uma significativa aprendizagem, independente do objeto de estudo que se deseja investigar e conhecer. No tocante à Astronomia. Amaral (2008) enfatiza que a Astronomia, como os demais campos de pesquisa científica, é fonte inesgotável de avanços, descobertas e revoluções tecnológicas. Trazendo elucidações dos "mistérios do universo". Tais situações favorecem um trabalho pedagógico contextualizador da ciência celeste em ambientes educacionais, apresentando-a como campo de estudo que constantemente revela novos conhecimentos.

Batista, Fontes e Pereira (2017) levantam as possibilidades de desenvolvimento que o ensino de Astronomia proporciona aos alunos:

\footnotetext{
Nesse sentido, a astronomia pode ser considerada um "motor" poderoso o suficiente para despertar a curiosidade pela ciência, para não somente desenvolve conceitos básicos, mas favorecer o desenvolvimento de outras características transversais à astronomia, tais como: melhoria na capacidade de cálculos matemáticos, comparação e classificação de objetos ou eventos, comunicação, experimentação, exploração, imaginação, medição, observação, organização, raciocínio lógico, aplicação, avaliação, dedução, descrição, interpretação, predição, manipulação de instrumentos e reconhecimento de pré-conceitos, ou concepções alternativas.
} 
A gama de possibilidades de desenvolvimentos de habilidades que o ensino de Astronomia propicia é relevante não apenas para o ensino de Física, mas também para outras áreas do conhecimento científico, que direta ou indiretamente utilizam os mesmos métodos científicos e didáticos para conhecer e explicar fenômenos e objetos astronômicos.

Observa-se que, para que a Astronomia possa ser um conteúdo aplicado no Ensino, com contribuições positivas, deve-se ter ciência da pertinência de seus conteúdos em relação com a realidade dos alunados e o atendimento das necessidades do seu contexto social. Como também possa levar ao aluno a compreender a noção de integração de todos os elementos e corpos existentes no universo.

Amaral (2008) salienta o fato de o ensino de Astronomia ser concatenado com outras frentes de estudo científico, além de explicitar as dificuldades encontradas no trabalho pedagógico.

Mas ensinar Astronomia não é simples. O indivíduo que se propõe a transmitir saberes astronômicos descobre-se numa tarefa astronômica, com o perdão do trocadilho. É um tema com abordagem multidisciplinar: história, geografia, filosofia, mitologia, física, química, matemática, biologia, geologia, meteorologia, cosmologia, entre outras. Exatamente por essa razão, o ensino de Astronomia é prejudicado, já que para realizá-lo é necessário dominar variadas áreas (AMARAL, 2008, p. 14)

Sendo assim, o ensino da Astronomia é algo pertinente e preciso para ser incluso em programas curriculares voltados aos alunos de diferentes níveis e modalidades de ensino, pois contribui para o desenvolvimento integral do educando, tanto em um trabalho interdisciplinar ou em uma abordagem multidisciplinar.

Os Parâmetros Curriculares Nacionais para o Ensino Médio de Ciências da Natureza, Matemática e suas Tecnologias (BRASIL, 2002), também conhecidos como PCN + de Ciências da Natureza, Matemática e suas Tecnologias, propõem que a Astronomia seja incluída, no componente curricular de Física, dentro do eixo temático "Terra, Universo e Vida", com ênfase em conhecimentos relacionados a: fatos e percepções científicas sobre a origem e evolução do Universo; compreensão de escalas de tempos cosmológicos; desenvolvimento e uso de equipamentos de astronomia observacional e radioastronomia; astrofísica estelar; além das possibilidades e condições de vida em outros astros no sistema solar e fora dele.

Contudo, não basta apenas que o $\mathrm{PCN}+$ recomende quais conteúdos relacionados à Astronomia serão inseridos no plano curricular no ensino de Física. É preciso levar em consideração a maneira como é abordado o assunto, relevando as dificuldades que alguns alunos da referida etapa escolar possuem em compreenderem e aplicarem as aprendizagens adquiridas.

Deter informações e instrumentação para o ensino de Astronomia é uma atribuição necessária para os docentes. Mesmo que o professor possua conhecimentos na área da 
Astronomia, e até experiência no ensino dos conteúdos do referido campo científico, faz-se necessário a utilização de materiais bibliográficos para facilitação dos processos de ensino e aprendizagem. Por isso, faz-se compreender a forma como os conteúdos astronômicos são abordados pelos livros didáticos de Física.

\section{II.2 Relevâncias do Livro Didático}

O estudo da Astronomia também requer que fontes de informação sejam utilizadas para que sua abordagem seja favorecida. O livro didático oportuniza, através da ação mediadora do docente entre os conhecimentos inseridos na bibliografia específica e os educandos, a apropriação dos saberes astronômicos. Sua função, nesse caso, é a de facilitar a aprendizagem dos alunos.

Existem pesquisas no meio acadêmico que analisaram como eram abordados os conteúdos astronômicos em livros didáticos, dentre eles: de Oliveira Coelho e Bulegon (2013); Batista, Fusinato e de Oliveira (2018); Langhi e Nardir (2007); Macedo, Dutra e Fernandes (2010); e Rhoden e Pauletti (2015). Contudo, esses trabalhos investigaram apenas materiais bibliográficos do PNLD do Ensino Fundamental, diferente do objetivo do trabalho de pesquisa desenvolvido e relatado neste artigo.

As vantagens que se podem obter com o uso do material didático são inúmeras. Contudo, há de se ter certo comedimento em querer aceitar e propagar todas as informações contidas neles. Conforme Langhi e Nardir (2007), existem casos em que materiais didáticos podem trazer informações e ilustrações erradas e/ou que possam dificultar a construção de aprendizagens astronômicas significativas.

É necessário ter ciência que nem todas as obras publicadas são uníssonas ao exporem um tema em questão. Leite e Housome (2007) comentam que as informações contidas em alguns materiais didáticos são conteúdos fragmentados, com pouca profundidade, quando não errôneos, e, ainda, consideravelmente insuficientes para a explicação das muitas questões veiculadas pelos meios de comunicação.

Já Bretones (1999) apresenta que há também a possibilidade de aceitação de teorias tacanhas dos conhecimentos celestes, tanto pelos professores quanto dos alunos, devido ao acesso de material didático inadequado para o ensino, instrumentos pedagógicos esses que podem contribuir para a formação de opinião tanto divergente quanto contraditória para com aquilo que realmente deve ser estudado e esclarecido sobre Astronomia. Muito embora haja uma avaliação criteriosa pelo Ministério da Educação e Cultura (MEC) dos livros didáticos que chegam às escolas públicas, é sabido que inúmeros exemplares são publicados e comercializados contendo erros conceituais ou até mesmo informações incompletas daquilo que abordam.

É preciso definir que o uso do livro didático não deva ir ao encontro da omissão docente no que se destina ao planejamento e criticidade dos conteúdos que serão abordados em sala de aula. O livro não é designado para substituir a ação do professor, que é a de 
ensinar. Ensino esse que ocorre através da mediação entre os educandos e os objetos de estudo. Um bom livro, na mão de um professor leigo, pode tornar o docente um mero propagador dos enunciados e proposta de trabalho do material didático adotado. Porém um professor bem formado e orientado faz uso do livro como aporte teórico, podendo até supera os limites estipulados pelos materiais didáticos.

\section{II.3 Erros conceituais em livros didáticos do PNLD 2018 de Física}

Um material bibliográfico bem elaborado, tanto em informações quanto em elementos visuais e icnográficos, é considerado um instrumento relevantemente satisfatório dentro de um trabalho didático-pedagógico. Serve como auxílio no trabalho docente de ensino, como também em uma fonte de consulta e levantamento de questões sobre temas específicos de um componente curricular ou área de conhecimento.

Lobato et al. (2009) destaca que atualmente muitos livros destinados à educação básica (especialmente para o Ensino Médio) estão apresentando seus conteúdos com linguagem mais simples e clara, com muitas referências em forma de imagens e esquemas, mapas conceituais, além de trazer questões interdisciplinares e projetos contextualizadores. De fato, há uma grande preocupação em relacionar os conteúdos ensinados com características do cotidiano e da realidade dos estudantes. Isso é muito comum nos atuais livros de Física ofertados às escolas públicas e privadas de ensino.

Contudo, mesmo com todo arcabouço teórico e seções no material didático que apresentam interface entre assuntos ligados à Física com outros campos (tecnologias, medicina, construções, transportes, por exemplo), percebe-se ainda alguns erros conceituais presentes em algumas dessas abordagens. Tratando-se do ensino de Astronomia, essa situação não foge a este diagnóstico. Langhi e Nardir (2007), em seu trabalho de pesquisa sobre incorreções conceituais sobre a Astronomia encontradas em livros didáticos analisados, elencaram os seguintes erros comumente encontrados nestes materiais de apoio educacional acerca dos fenômenos astronômicos e seus astros: as causas das estações do ano; as fases lunares; os movimentos do Planeta Terra; a definição de constelações, representações gráficas dos astros e suas órbitas, e até sobre a história da construção do conhecimento celeste.

Desta forma, fica claro que o profissional da educação responsável pelo ensino de Astronomia também precisa ficar atento sobre a veracidade dos dados e as representações iconográficas factuais dos materiais bibliográficos que possui, a fim de evitar a propagação de informações equivocadas sobre corpos celestes e fenômenos astronômicos.

\section{Metodologia}

No presente estudo foi utilizada a pesquisa de caráter bibliográfico, utilizando o procedimento de análise dos conteúdos, metodologia proposta por Bardin (1995), tendo como 
objetivo de verificar quais e quantos conteúdos presentes nos referenciais analisados com parâmetros e orientações curriculares presentes nos documentos oficiais.

Foram analisadas obras didáticas ofertadas pelo Programa Nacional do Livro e do Material Didático (PNLD), para o Triênio 2018-2020, para a disciplina de Física no Ensino Médio. No total, foram doze coleções analisadas, cada um com três livros (um por série), em que se buscou encontrar informações e abordagens relativas ao ensino de Astronomia em cada obra.

Para uma melhor orientação do processo investigativo, foram utilizados norteamentos presentes nos Parâmetros Curriculares Nacionais para o Ensino Médio (Brasil, 2010), e nas Orientações Curriculares para o Ensino Médio (Brasil, 2006), sobre como a disciplina de Física pode abordar conteúdos astronômicos em suas propostas curriculares. Também foi incluído no processo de análise das obras sugestões de temas e conteúdos presentes na obra de Oliveira Filho e Saraiva (2004).

As coleções didáticas avaliadas, e que constam no PNLD 2018, são Carron, Guimarães e Piqueira (2016); Gaspar (2016); Luz, Álvares e Guimarães (2016); Fukui et al. (2016); Fuke e Yamamoto (2016); Gaulter, Helou e Newton (2016); Gonçalves Filho e Toscano (2016); Barreto e Xavier (2016); Bonjorno et al. (2016); Pietrocola et al. (2016); Torres et al. (2016); Martini et al. (2016).

$\mathrm{Na}$ atividade analítica dos materiais didáticos, determinados conteúdos e temas foram elencados para avaliar a abrangência das abordagens presentes nos livros sobre fenômenos astronômicos e suas relações com conhecimentos físicos e nos demais campos científicos, sócio-históricos e culturais da humanidade. Segue as Tabelas contendo os dados coletados e organizados conforme os critérios avaliativos adotados, além das referências das obras analisadas, classificados em três eixos, conforme orientações dos Parâmetros Curriculares Nacionais para o Ensino Médio (Brasil, 2010): Terra e o Sistema Solar, O Universo e Sua Origem, e Compreensão Humana do Universo.

Tabela 1 - Eixos temáticos e assuntos sobre a Astronomia, no PCN + de Ciências Naturais.

\begin{tabular}{|c|c|}
\hline EIXOS TEMÁTICOS & ASSUNTOS/CONTEÚDOS \\
\hline \multirow{5}{*}{$\begin{array}{l}\text { A - TERRA E O } \\
\text { SISTEMA SOLAR }\end{array}$} & $\begin{array}{l}\text { 1- Relações entre os movimentos da Terra, da Lua e do Sol para a descrição de } \\
\text { fenômenos astronômicos (período de duração do dia e da noite, as quatro estações } \\
\text { do ano, as quatro fases da lua, eclipses solar e lunar). }\end{array}$ \\
\hline & 2- Abordagem sobre o movimento de precessão do Eixo de rotação da Terra. \\
\hline & $\begin{array}{l}\text { 3- Compreender as interações gravitacionais entre corpos celestes forças e relações } \\
\text { de conservação no movimento dos corpos celestes }\end{array}$ \\
\hline & 4- Abordagem sobre Efeito das marés. \\
\hline & $\begin{array}{l}\text { 5- Explicações sobre a mecânica orbital dos corpos menores do Sistema Solar } \\
\text { (Asteroides, cometas e planetoides). }\end{array}$ \\
\hline
\end{tabular}




\begin{tabular}{|c|c|}
\hline & $\begin{array}{l}\text { 6- Apresenta a correta representação ou referência da excentricidade orbital dos } \\
\text { planetas do Sistema Solar. }\end{array}$ \\
\hline \multirow{6}{*}{$\begin{array}{l}\text { B - O UNIVERSO E } \\
\text { SUA ORIGEM }\end{array}$} & $\begin{array}{l}\text { 1- Apresentação das teorias científicas que explicam a origem, evolução e } \\
\text { constituição do Universo. }\end{array}$ \\
\hline & $\begin{array}{l}\text { 2- Abordagem das atuais formas de investigação dos fenômenos celestes e da } \\
\text { origem e evolução do Cosmos. }\end{array}$ \\
\hline & $\begin{array}{l}\text { 3- Compreender a distribuição dos astros e suas formas de organização no } \\
\text { Universo. }\end{array}$ \\
\hline & 4- Reconhecer ordens de grandeza de medidas astronômicas. \\
\hline & 5- Situar a vida humana no tempo e no espaço cosmológico. \\
\hline & 6- Discutir as hipóteses de existência e/ou condições de vida fora da Terra. \\
\hline \multirow{3}{*}{$\begin{array}{c}\text { C - COMPREENSÃO } \\
\text { HUMANA DO } \\
\text { UNIVERSO }\end{array}$} & $\begin{array}{l}\text { 1- Conhecer aspectos dos modelos explicativos da origem e constituição do } \\
\text { Universo, segundo diferentes culturas. }\end{array}$ \\
\hline & $\begin{array}{l}\text { 2- Compreender aspectos da evolução dos modelos da ciência para explicar a } \\
\text { constituição do Universo, através dos tempos. }\end{array}$ \\
\hline & $\begin{array}{l}\text { 3- Identificar diferentes formas pelas quais os modelos explicativos do Universo } \\
\text { influenciaram a cultura e a vida humana ao longo da história da humanidade. }\end{array}$ \\
\hline
\end{tabular}

Tabela 2 - Relação entre as Coleções de Física do PNLD 2018 com os assuntos dos eixos temáticos sobre Astronomia do PCN + de Ciências Naturais (Tabela 1).

\begin{tabular}{|c|c|c|c|c|c|c|}
\hline $\mathbf{N}$ & $\begin{array}{c}\text { Título do } \\
\text { Livro }\end{array}$ & Autor & Editora & $\begin{array}{c}\text { Código do } \\
\text { PNLD2018 } \\
\end{array}$ & $\begin{array}{l}\text { Conteúdos dos } \\
\text { eixos temáticos }\end{array}$ & $\begin{array}{c}\text { TOTAL e } \\
(\%) \\
\end{array}$ \\
\hline 1 & $\begin{array}{c}\text { Física: } \\
\text { Contexto \& } \\
\text { Aplicações }\end{array}$ & $\begin{array}{l}\text { Luz, Álvares e } \\
\text { Guimarães }\end{array}$ & Scipione & $0045 \mathrm{P} 1813$ & $\begin{array}{c}\mathrm{A} 1, \mathrm{~A} 2, \mathrm{~A} 3, \mathrm{~A} 4, \mathrm{~A} 5, \\
\mathrm{~A} 6, \mathrm{~B} 1, \mathrm{~B} 2, \mathrm{~B} 3, \mathrm{~B} 4, \\
\mathrm{~B} 5, \mathrm{C} 1, \mathrm{C} 2, \mathrm{C} 3\end{array}$ & $14(93,33)$ \\
\hline 2 & $\begin{array}{l}\text { Física em } \\
\text { Contextos }\end{array}$ & Pietrocola et al. & $\begin{array}{c}\text { Editora do } \\
\text { Brasil }\end{array}$ & 0167P18133 & $\begin{array}{c}\mathrm{A} 1, \mathrm{~A} 3, \mathrm{~A} 4, \mathrm{~A} 5, \mathrm{~A} 6, \\
\mathrm{~B} 1, \mathrm{~B} 2, \mathrm{~B} 3, \mathrm{~B} 4, \mathrm{~B} 5, \\
\mathrm{C} 1, \mathrm{C} 2, \mathrm{C} 3\end{array}$ & $13(86,67)$ \\
\hline 3 & $\begin{array}{c}\text { Física - } \\
\text { Ciência e } \\
\text { Tecnologia }\end{array}$ & Torres et al. & Moderna & 0188P18133 & $\begin{array}{c}\mathrm{A} 1, \mathrm{~A} 3, \mathrm{~A} 5, \mathrm{~A} 6, \mathrm{~B} 1, \\
\mathrm{~B} 2, \mathrm{~B} 3, \mathrm{~B} 4, \mathrm{~B} 5, \mathrm{C} 1, \\
\mathrm{C} 2, \mathrm{C} 3\end{array}$ & $12(80,00)$ \\
\hline 4 & Física & $\begin{array}{l}\text { Gaulter, Helou } \\
\text { e Newton }\end{array}$ & Saraiva & 0101P18133 & $\begin{array}{c}\mathrm{A} 1, \mathrm{~A} 3, \mathrm{~A} 5, \mathrm{~A} 6, \mathrm{~B} 1, \\
\mathrm{~B} 2, \mathrm{~B} 3, \mathrm{~B} 4, \mathrm{~B} 5, \mathrm{C} 1, \\
\mathrm{C} 2, \mathrm{C} 3\end{array}$ & $12(80,00)$ \\
\hline 5 & Física & Bonjorno et al. & FTD & 0131P18133 & $\begin{array}{c}\mathrm{A} 1, \mathrm{~A} 3, \mathrm{~A} 6, \mathrm{~B} 1, \mathrm{~B} 2, \\
\mathrm{~B} 5, \mathrm{C} 1, \mathrm{C} 2, \mathrm{C} 3\end{array}$ & $09(60,00)$ \\
\hline 6 & Física & $\begin{array}{c}\text { Carron, } \\
\text { Guimarães e }\end{array}$ & Ática & $0021 \mathrm{P} 18133$ & $\begin{array}{c}\mathrm{A} 1, \mathrm{~A} 3, \mathrm{~B} 1, \mathrm{~B} 2, \mathrm{~B} 3, \\
\mathrm{~B} 4, \mathrm{C} 1, \mathrm{C} 2, \mathrm{C} 3\end{array}$ & $09(60,00)$ \\
\hline
\end{tabular}




\begin{tabular}{|c|c|c|c|c|c|c|}
\hline & & Piqueira & & & & \\
\hline 7 & $\begin{array}{c}\text { Ser } \\
\text { Protagonista }\end{array}$ & Fukui et al. & SM & 0071P18133 & $\begin{array}{c}\mathrm{A} 1, \mathrm{~A} 3, \mathrm{~B} 1, \mathrm{~B} 2, \mathrm{~B} 3, \\
\mathrm{~B} 4, \mathrm{~B} 5, \mathrm{C} 2, \mathrm{C} 3\end{array}$ & $09(60,00)$ \\
\hline 8 & $\begin{array}{l}\text { Física Aula } \\
\text { por aula }\end{array}$ & $\begin{array}{l}\text { Barreto e } \\
\text { Xavier }\end{array}$ & FTD & 0129P18133 & $\begin{array}{c}\mathrm{A} 1, \mathrm{~A} 3, \mathrm{~A} 6, \mathrm{~B} 2, \mathrm{~B} 5 \\
\mathrm{C} 1, \mathrm{C} 2, \mathrm{C} 3\end{array}$ & $08(53,33)$ \\
\hline 9 & $\begin{array}{c}\text { Compreendend } \\
\text { o a Física }\end{array}$ & Alberto Gaspar & Ática & 0025P18133 & $\begin{array}{c}\mathrm{A} 1, \mathrm{~A} 3, \mathrm{~A} 6, \mathrm{~B} 2, \mathrm{~B} 4, \\
\mathrm{C} 1, \mathrm{C} 2, \mathrm{C} 3\end{array}$ & $08(53,33)$ \\
\hline 10 & $\begin{array}{l}\text { Física para o } \\
\text { Ensino Médio }\end{array}$ & $\begin{array}{c}\text { Fuke e } \\
\text { Yamamoto }\end{array}$ & Saraiva & 0100P18133 & $\begin{array}{c}\mathrm{A} 1, \mathrm{~A} 2, \mathrm{~A} 3, \mathrm{~B} 4, \mathrm{~B} 5, \\
\mathrm{C} 2, \mathrm{C} 3\end{array}$ & 07() \\
\hline 11 & $\begin{array}{c}\text { Física: } \\
\text { Interação e } \\
\text { Tecnologia }\end{array}$ & $\begin{array}{c}\text { Gonçalves } \\
\text { Filho e } \\
\text { Toscano }\end{array}$ & Leya & 0118P18133 & $\begin{array}{c}\mathrm{A} 1, \mathrm{~A} 3, \mathrm{~B} 2, \mathrm{~B} 4, \mathrm{C} 2, \\
\mathrm{C} 3\end{array}$ & 06 \\
\hline 12 & $\begin{array}{c}\text { Conexões com } \\
\text { a Física }\end{array}$ & Martini et al. & Moderna & 0200P18133 & $\mathrm{A} 1, \mathrm{~A} 3, \mathrm{~A} 6, \mathrm{~B} 1, \mathrm{C} 1$ & 05 \\
\hline
\end{tabular}

\section{Resultados e discussões}

Os materiais didáticos analisados apresentaram dados relevantes sobre como os fenômenos e corpos astronômicos são abordados dentro da disciplina de Física. Constata-se que conteúdos astronômicos foram encontrados em cada coleção analisada, tanto através de informações presentes nos conteúdos da disciplina quanto em questões/exercícios tratando sobre fenômenos astronômicos ou corpos celestes. Porém, percebe-se que nem todos os assuntos propostos pelos eixos temáticos sobre Astronomia estão presentes em cada coleção didática analisada.

Em relação à quantidade de assuntos, percebe-se a diferença entre as quantidades de conteúdos específicos da Astronomia abordados entre as coleções. Dentre elas, a coleção do autor Luz, Álvares e Guimarães é a que mais apresenta os elementos astronômicos, (14 conteúdos, de um total de 15), seguida da coleção de Pietrocola et al. A que menos trata assuntos astronômicos em seu material didático é a coleção de Martini et al., com apenas 5 pontos do eixo temático sobre Astronomia do PCN + exposto em seus livros. Ainda sobre a obra de Martini et al., ela apresentou apenas um conteúdo acerca do eixo "O Universo e Sua Origem", como também só trata de um conteúdo sobre a "Compreensão Humana do Universo".

Coincidentemente, as obras de Torres et al. e Gaulter, Helou e Newton, como as de Carron, Guimarães e Piqueira (0021P18133) e Fukui et al. (0071P18133), possuem as mesmas abordagens de conteúdos astronômicos. Na primeira dupla, os doze assuntos presentes em cada obra são os mesmos. Vale salientar que essas obras são de editoras distintas, e não compartilham os mesmos autores. 
Conforme mostra a Tabela 2, as abordagens sobre o movimento de precessão do eixo da Terra, o efeito de maré das águas oceânicas e os estudos sobre os corpos menores do sistema solar estão presentes em menos da metade das obras avaliadas. Isso dificulta a elucidação da dinâmica dos movimentos terrestres, da relação das forças gravitacional da Lua e do Sol sobre as partículas mais leves do nosso planeta, e a diferenciação entre os astros que formam o Sistema Solar, respectivamente.

Também é constatado que a abordagem correta sobre a excentricidade das órbitas elípticas dos planetas não é algo presente em todas as obras. Quatro delas sequer cita o grau de achatamento orbital dos planetas do Sistema Solar, e é quase uma unanimidade na representação gráfica do movimento translacional dos referidos astros órbitas demasiadamente excêntricas. Direta e/ou indiretamente, pode-se passar noções erradas do movimento de translação, do apogeu e perigeu dos astros, variação de temperatura no planeta e até mesmo das estações do ano na Terra.

Sobre os dados analisados no eixo "O Universo e Sua Origem", cinco das doze coleções estudadas não trazem teorias que explicam o surgimento do universo e desenvolvimento do cosmos. Seis obras não citam como os astros estão organizados no Universo, três materiais bibliográficos não abordam unidades de medidas de grandezas astronômicas, e nenhum livro debate ou problematiza hipóteses e/ou condições para o surgimento e desenvolvimento de vida fora da Terra. Pelo fato do próprio eixo temático do $\mathrm{PCN}+$ de Ciências Naturais destacar a necessidade da abordagem de teorias científicas acerca da origem e desenvolvimento do Cosmos, deixar de destacar esses conhecimentos nos livros de Física provoca estranhamento.

Em relação ao eixo "Compreensão Humana do Universo", três coleções analisadas não abordavam as explicações sobre a origem do cosmos na perspectiva de diferentes povos e culturas. Mesmo que o foco de todos os materiais seja a apresentação de conteúdos e conhecimentos comprovados e aceitos na comunidade científica, a Etnoastronomia tem sua importância no contexto histórico e cultural da construção do conhecimento humano, através da interpretação e descrições que diferentes povos desenvolveram sobre os astros e fenômenos celestes.

$\mathrm{Na}$ análise dos dados coletados em cada obra, como também na comparação entre as coleções que foram objetos de estudo deste trabalho de pesquisa, constata-se que as obras disponibilizadas pelo PNLD 2018 não são consonantes em relação a quantidade de assuntos astronômicos que o PCN + de Ciências Naturais especifica para o Ensino Médio. Isso mostra que, dependendo dos autores e da forma de abordagem dos conteúdos, temas astronômicos podem ou não ser abordados em outras seções dos materiais didáticos, além dos segmentos mais comuns para todos: gravitação universal e física moderna.

Mesmo que a proposta principal dos livros didáticos analisados seja a divulgação de conhecimentos gerais do campo da Física no Ensino Médio, tanto como instrumentos de auxílio docente quanto na condição de materiais facilitadores de aprendizagens por parte dos 
estudantes, deixar de abordar determinados assuntos astronômicos, propostos pelo $\mathrm{PCN}+\mathrm{de}$ Ciências Naturais, compromete a aprendizagem de leis e teorias científicas específicas da Física e/ou o entendimento das causa e consequências de determinados fenômenos naturais, caso o docente utilize o livro didático como principal fonte de referência e roteiro de planejamento de ensino nas suas aulas.

Dentro do trabalho de pesquisa e investigação, também foram feitas comparações entre a quantidade de obras didáticas adotadas no país, nas unidades federativas, nos municípios, e nos estabelecimentos de ensino brasileiros, atendidos pelo PNLD 2018. As informações citadas anteriormente constam na Tabela 3.

Tabela 3 - Quantidade de exemplares das obras do PNLD 2018 de Física distribuídos.

\begin{tabular}{|c|c|c|c|c|c|}
\hline $\begin{array}{c}\text { Código das } \\
\text { coleções }\end{array}$ & Autor & $\begin{array}{c}\text { Quantidade de } \\
\text { exemplares } \\
\text { distribuídos BR }\end{array}$ & $\begin{array}{l}\text { Quantidade de } \\
\text { estados cujo } \\
\text { livro é o mais } \\
\text { adotado }\end{array}$ & $\begin{array}{c}\text { Quantidade de } \\
\text { municípios cujo } \\
\text { livro é o mais } \\
\text { adotado }\end{array}$ & $\begin{array}{l}\text { Quantidade de } \\
\text { escolas cujo } \\
\text { livro é adotado }\end{array}$ \\
\hline 0021P1813310 & $\begin{array}{l}\text { Carron, } \\
\text { Guimarães } \\
\text { e Piqueira }\end{array}$ & 228720 & 0 & 139 & 604 \\
\hline 0025P1813310 & $\begin{array}{l}\text { Alberto } \\
\text { Gaspar }\end{array}$ & 279887 & 0 & 170 & 887 \\
\hline 0118P1813310 & $\begin{array}{c}\text { Gonçalves } \\
\text { Filho e } \\
\text { Toscano }\end{array}$ & 365058 & 0 & 271 & 1174 \\
\hline 0167P1813310 & $\begin{array}{c}\text { Pietrocola } \\
\text { et al. }\end{array}$ & 394836 & 1 & 190 & 891 \\
\hline 0045P1813310 & $\begin{array}{c}\text { Luz, } \\
\text { Álvares e } \\
\text { Guimarães }\end{array}$ & 527707 & 0 & 394 & 1456 \\
\hline 0188P1813310 & $\begin{array}{c}\text { Torres et } \\
\text { al. }\end{array}$ & 559153 & 0 & 330 & 1546 \\
\hline 0101P1813310 & $\begin{array}{l}\text { Gaulter, } \\
\text { Helou e } \\
\text { Newton }\end{array}$ & 611075 & 0 & 336 & 1529 \\
\hline 0100P1813310 & $\begin{array}{c}\text { Fuke e } \\
\text { Yamamoto }\end{array}$ & 689022 & 1 & 395 & 1650 \\
\hline 0200P1813310 & $\begin{array}{c}\text { Martini et } \\
\text { al. }\end{array}$ & 691363 & 1 & 465 & 1892 \\
\hline 0071P1813310 & Fukui et & 766948 & 0 & 460 & 1915 \\
\hline
\end{tabular}




\begin{tabular}{|c|c|c|c|c|c|}
\hline & al. & & & & \\
\hline $\mathbf{0 1 2 9 P 1 8 1 3 3 1 0}$ & $\begin{array}{c}\text { Barreto e } \\
\text { Xavier }\end{array}$ & 971169 & 3 & 728 & 2490 \\
\hline $\mathbf{0 1 3 1 P 1 8 1 3 3 1 0}$ & $\begin{array}{c}\text { Bonjorno } \\
\text { et al. }\end{array}$ & 1518533 & 21 & 1385 & 4253 \\
\hline
\end{tabular}

Fonte: PNLD.

Em uma correlação dos dados presentes na Tabela 2 e na Tabela 3, pode-se comparar as obras analisadas com suas respectivas distribuições nos níveis nacional, estadual, municipal e nos estabelecimentos de ensino. Em uma primeira constatação, percebe-se que a obra 0045P1813 (LUZ; ÁLVARES; GUIMARÃES, 2016) que mais apresentou abordagens astronômicas em seus livros, não foi a mais adotada no PNLD 2018. A coleção anteriormente citada foi a $8^{a}$ colocada a nível nacional e entre as escolas, além de ocupar a $6^{\text {a }}$ posição das escolhas no nível municipal. A coleção de Bonjorno et al. (2016), com apenas 9 (nove) abordagens detectadas sobre à Astronomia em sua bibliografia, foi o material didático mais distribuído em todos os níveis.

$\mathrm{O}$ segundo material mais adotado em todos os níveis pesquisados foi o 0129P1813310, de Barreto e Xavier (2016), conforme consta na Tabela 3. Já na tabela 2, o mesmo material está entre o cinco que menos apresentaram abordagens astronômicas em seus livros didáticos. A coleção 0200P1813310 de Martini et al. (2016), com menor quantidade de conteúdos astronômicos abordados, em comparação as demais coleções avaliadas da Tabela 2 , foi o $4^{\circ}$ material didático de Física mais distribuído pelo país e entre as escolas, além de ter sido a $3^{\text {a }}$ coleção mais escolhida no comparativo entre estados e municípios.

Já a obra de Carron, Guimarães e Piqueira (2016), com código 0021P1813310, foi a coleção menos escolhida em todos os níveis da Tabela 3. Contudo, a mesma ficou entre a primeira metade das obras que mais abordam assuntos astronômicos, conforme consta na Tabela 2.

Através das comparações feitas entre os dados presentes nas Tabelas 2 e 3, constatase que os materiais mais distribuídos pelo PNLD 2018 não são aqueles que trouxeram mais abordagens astronômicas em suas obras. E pela leitura que se faz do comparativo entre as perspectivas presentes nas tabelas, percebe-se que a quantidade de assuntos/conteúdos astronômicos não foi um fator de grande peso na escolha dos livros didáticos.

Essas constatações feitas na comparação entre a quantidade de obras avaliadas pela quantidade de assuntos astronômicos (Tabela 1) e suas distribuições pelo PNLD 2018 (Tabela 3) não desmerecem as escolhas feitas pelos professores e suas redes de ensino, e nem são observações que visam criticar as opções adotadas nos níveis nacional, estaduais, municipais e pela maioria das escolas. Essas informações enriqueceram o trabalho de pesquisa, possibilitando entender se o atendimento aos eixos temáticos sobre Astronomia do $\mathrm{PCN}+\mathrm{de}$ 
Ciências Naturais, nas coleções de Física para o Ensino Médio, foram ou não relevantes na decisão e suas adoções nos estabelecimentos de ensino.

Outra comparação que pode ser feita diz respeito a quantidade de coleções que apresentam os conteúdos relacionados a astronomia, conforme indicados na Tabela 2. A Fig. 1 mostra a quantidade percentual de coleções que contempla os conteúdos. Os itens A1 e A3 estão disponíveis em todas as 12 coleções. Já o item B6 não é apresentado em nenhuma das obras do PNLD 2018, como este item, conforme Tabela 1, consiste em "Discutir as hipóteses de existência e/ou condições de vida fora da Terra", que é um tema abrangente e interdisciplinar, é possível que os autores tenham dificuldades de relacioná-lo com os demais tópicos da Física.

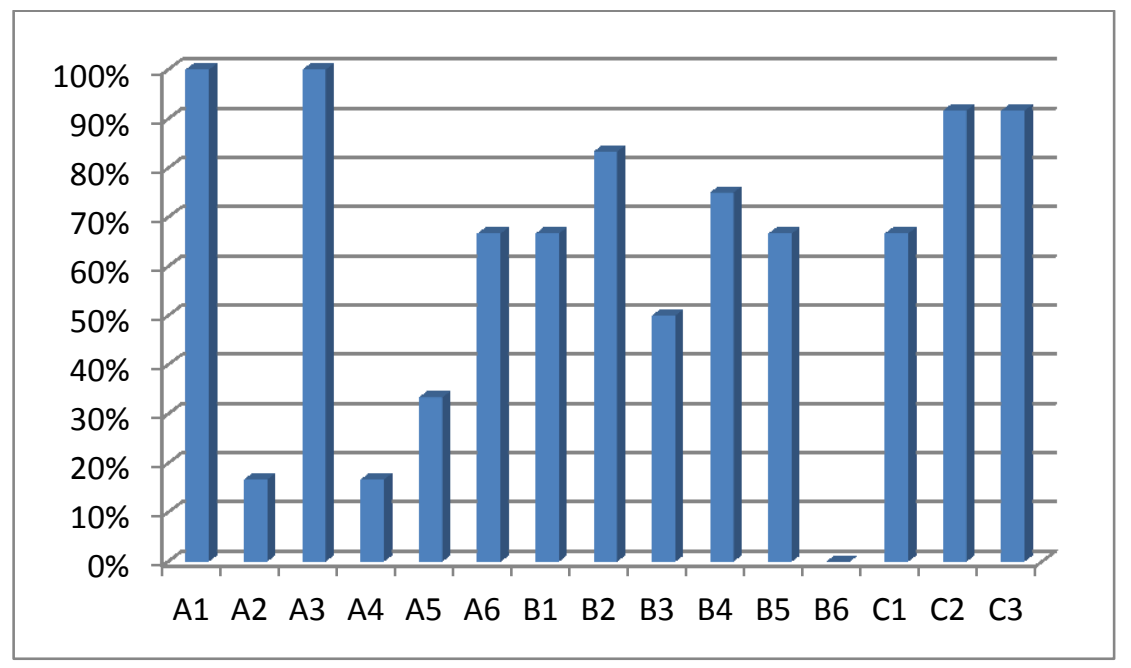

Fig. 1 - Quantidade de obras que contemplam cada um dos itens dos $\mathrm{PCN}+$ de sobre astronomia.

\section{Conclusão}

A Astronomia é um campo de conhecimento bastante amplo, que possibilita até mesmo que outras ciências utilizem seus conhecimentos para elucidar situações-problemas que as mesmas possuem, e interpretar fenômenos astronômicos que interferem em seus objetos de estudo. As relações que os astros têm com o cotidiano é algo importante, e conhecer o Universo é um dos caminhos para a humanidade compreender seus lugar e situação no cosmos.

Os conhecimentos sobre o Cosmos, nos dias atuais, têm se tornado mais compreensíveis e acessíveis do que em tempos mais remotos, períodos esses que requeriam dos estudiosos mais esforços e perseverança para superarem os obstáculos oriundos de diversas esferas (instrumentais, científico, religioso). Atualmente, as utilizações de diferentes referências bibliográficas através dos meios de comunicação e difusão das informações 
facilitam a aquisição de conhecimentos astronômicos. Neste caso, o livro didático é um instrumento que, dentro dos conteúdos que o mesmo aborda, tem o papel de facilitador do ensino da Astronomia.

A atividade de pesquisa das coleções disponibilizadas pelo PNLD 2018 mostrou que todas as obras apresentaram conteúdos astronômicos em seus livros. Contundo, ficou evidente que nem todas abordam todos os assuntos especificados pelos eixos temáticos do $\mathrm{PCN}+$ de Ciências Naturais. Mesmo que os eixos temáticos sobre tópicos astronômicos, presentes nos $\mathrm{PCN}+$, tratem especificamente dos temas que os docentes de Física precisam ministrar em suas aulas, é necessário que os materiais didáticos que auxiliam os professores e alunos contenham o máximo possível de informações e conteúdos sobre a Astronomia, a fim de que estes possam auxiliar cada vez mais aos processos de ensino e aprendizagem sobre o Cosmos, seus fenômenos e elementos.

Através de pesquisas bibliográficas e, consequentemente, reflexões acerca da forma como vem sendo tratado o ensino astronômico em livros de Física destinado à última etapa da educação básica nesse trabalho científico, constata-se a necessidade de boa parte destes materiais didáticos inserirem mais conteúdos sobre Astronomia e/ou reformularem a maneira como estão abordando os conhecimentos de uma ciência que, a cada dia, vem exigindo e expondo sua relevância como ramo científico necessário para a sociedade humana.

\section{Referências bibliográficas}

AMARAL, P. O ensino de astronomia nas séries finais do ensino fundamental: uma proposta de material didático de apoio ao professor. 2008. Dissertação (Mestrado) - Programa de Pós-Graduação de Ensino de Ciências, Instituto de Física, Universidade de Brasília, Brasília.

BARDIN, L. Análise de conteúdo. Lisboa: Edições 70, 1995.

BARRETO, B.; XAVIER, C. Física aula por aula. São Paulo: FTD, 2010.

BATISTA, M. C.; FONTES, A. D. S.; PEREIRA, R. F. Ensino de Astronomia: o problema da órbita da Terra. Arquivos do Museu Dinâmico Interdisciplinar da Universidade Estadual de Maringá, v. 21, n. 3, p. 155-165, 2017.

BATISTA, M. C.; FUSINATO, P. A.; DE OLIVEIRA, A. A. Astronomia nos livros didáticos de Ciências do Ensino Fundamental I. Ensino \& Pesquisa, 2018.

BONJORNO, J. R. et al. Física. 3. ed. São Paulo: FTD, 2016. 
BRASIL, M. E. C. Parâmetros curriculares nacionais: ensino médio. Brasília: Ministério da Educação, 2010.

BRASIL, M. E. C. SEB. Orientações Curriculares para o Ensino Médio. Ciências da Natureza, Matemática e suas Tecnologias. Brasília: MEC, SEB, 2006.

BRASIL. MINISTÉRIO DA EDUCAÇÃO. Secretaria de Educação Média e Tecnológica. PCN+ Ensino médio: orientações educacionais complementares aos Parâmetros Curriculares Nacionais-Ciências da Natureza, Matemática e suas Tecnologias. 2002.

BRETONES, P. S. Disciplinas introdutórias de astronomia nos cursos superiores do Brasil. 1999. 187p. Dissertação (Mestrado) - Universidade Estadual de Campinas, Instituto de Geociencias, Campinas, SP. Disponível em:

$<$ http://www.repositorio.unicamp.br/handle/REPOSIP/287056>.

DE OLIVEIRA COELHO, F. B.; BULEGON, A. M. Análise do tema Astronomia, nos livros didáticos indicados pelo PNLD, dos anos iniciais do Ensino Fundamental. Vidya, v. 33, n. 1, p. 12, 2013.

FREIRE, P. Pedagogia da autonomia: saberes necessários à prática educativa. São Paulo: Paz e Terra, 1996.

FUKUI, A.; MOLINA, M. D. M.; VENÊ; NANI, A. P. S. N. Ser Protagonista: Física. 3. ed. São Paulo: Editora SM, LTDA, 2016.

GASPAR, A. Compreendendo a Física. Editora Ática, 2016.

GONÇALVES FILHO, A.; TOSCANO, C. Física: interação e tecnologia. São Paulo: Leya, 2016.

GUALTER, J. B.; NEWTON, V. B.; HELOU, R. D. Tópicos de Física. São Paulo: Saraiva, 2016.

GUIMARÃES, O.; PIQUEIRA, J. R.; CARRON, W. Física. São Paulo: Ática, 2016.

LANGHI, R.; NARDI, R. Ensino de Astronomia: Erros conceituais mais comuns presente em livros didáticos de ciência. Caderno Brasileiro de Ensino de Física, v. 24, n. 1, p. 87-111, 2007. 
LANGHI, R.; NARDI, R. Justificativas para o ensino de Astronomia: o que dizem os pesquisadores brasileiros? Revista Brasileira de Pesquisa em Educação em Ciências, v. 14, n. 3, p. 041-059, 2014.

LANGHI, R.; NARDI, R. Ensino da astronomia no Brasil: educação formal, informal, não formal e divulgação científica. Revista Brasileira de Ensino de Física, v. 31, n. 4, p. 44024412, 2009.

LEITE, C.; HOSOUME, Y. Os professores de ciências e suas formas de pensar a astronomia. Revista Latino-Americana de Educação em Astronomia, n. 4, p. 47-68, 2007.

LOBATO, A. C. et al. Dirigindo o olhar para o efeito estufa nos livros didáticos de ensino médio: é simples entender esse fenômeno? Ensaio Pesquisa em Educação em Ciências, v. 11, n. 1, p. 7-22, 2009.

LUZ, A. M. R. D.; ÁLVARES, B. A.; GUIMARÃES, C. D. C. Física: contextos e aplicações. 2. ed. São Paulo: Scipione, 2016.

MACEDO, E. S.; DUTRA, G.; FERNANDES, S. O Ensino de Astronomia em Amargosa: uma reflexão sobre os livros didáticos utilizados no município. In: ENCONTRO NACIONAL DE ASTRONOMIA, n. 4, 2010, Recife. Disponível em:

$<$ http://www.sociedadeastronomica.com.br/enast/trabalhos/ASTRONOMIA_NOS_LIVROS_ DIDATICOS_EM_AMARGOSA.pdf $>$. Acesso em: 05 out. 2018.

MARTINI, G. et al. Conexões com a Física. São Paulo: Moderna, 2016.

OLIVEIRA FILHO, K. D. S.; SARAIVA, M. D. F. O. Astronomia e astrofísica. São Paulo: Livraria da Física, 2004.

PIETROCOLA, M. et al. Física em contextos: pessoal, social e histórico. São Paulo: Editora do Brasil, 2016.

RHODEN, F. H.; PAULETTI, D. Análise conceitual e didática dos conteúdos de Astronomia apresentados em livros de ensino fundamental, 2015. Disponível em:

$<$ https://rd.uffs.edu.br/bitstream/prefix/603/1/RHODEN.pdf>. Acesso em: 01 out. 2018.

TORRES, C. M. A. et al. Física: ciência e tecnologia. 4. ed. São Paulo: Moderna, 2016. 
YAMAMOTO, K.; FUKE, L. F. Física para o Ensino Médio. 4. ed. São Paulo: Saraiva, 2016.

BRASIL. MEC - Ministério da Educação. Secretaria de Educação Fundamental. Programa Nacional do Livro Didático 2004. Guia de livros didáticos $\mathbf{1}^{\mathbf{a}}$ a $\mathbf{4}^{\mathbf{a}}$ Séries. v. 2. 275p. Brasília: MEC, 2003. Disponível em:

$<$ https://www.fnde.gov.br/index.php/centrais-de-conteudos/publicacoes/category/125guias?download=9012: guia-pnld-2004>. Acesso em: 20 jun. 2020. 